\title{
Coenzyme Q10 levels are low and may be associated with the inflammatory cascade in septic shock
}

\author{
Michael W Donnino ${ }^{1,2^{*}}$, Michael N Cocchi ${ }^{1,3}$, Justin D Salciccioli ${ }^{1}$, Daniel Kim ${ }^{1}$, Ali B Naini ${ }^{4}$, Catherine Buettner ${ }^{5}$ \\ and Praveen Akuthota ${ }^{2,6}$
}

\begin{abstract}
Introduction: Mitochondrial dysfunction is associated with increased mortality in septic shock. Coenzyme Q10 (CoQ10) is a key cofactor in the mitochondrial respiratory chain, but whether CoQ10 is depleted in septic shock remains unknown. Moreover, statin therapy may decrease CoQ10 levels, but whether this occurs acutely remains unknown. We measured CoQ10 levels in septic shock patients enrolled in a randomized trial of simvastatin versus placebo.

Methods: We conducted a post hoc analysis of a prospective, randomized trial of simvastatin versus placebo in patients with septic shock (ClinicalTrials.gov ID: NCT00676897). Adult patients with suspected or confirmed infection and the need for vasopressor support were included in the initial trial. For the current analysis, blood specimens were analyzed for plasma CoQ10 and low-density lipoprotein (LDL) levels. The relationship between CoQ10 levels and inflammatory and vascular endothelial biomarkers was assessed using either the Pearson or Spearman correlation coefficient.
\end{abstract}

Results: We analyzed 28 samples from 14 patients. CoQ10 levels were low, with a median of 0.49 (interquartile range 0.26 to 0.62$)$ compared to levels in healthy control patients $(\mathrm{CoQ} 10=0.95 \mu \mathrm{mol} / \mathrm{L} \pm 0.29 ; P<0.0001)$. Statin therapy had no effect on plasma CoQ10 levels over time $(P=0.13)$. There was a statistically significant relationship between plasma CoQ10 levels and levels of vascular cell adhesion molecule (VCAM) $\left(r^{2}=0.2 ; P=0.008\right)$, TNF- $\alpha\left(r^{2}\right.$ $=0.28 ; P=0.004), I L-8\left(r^{2}=0.21 ; P=0.015\right), I L-10\left(r^{2}=0.18 ; P=0.025\right)$, E-selectin $\left(r^{2}=0.17 ; P=-0.03\right), I L-1 r a\left(r^{2}=\right.$ $0.21 ; P=0.014), I L-6\left(r^{2}=0.17 ; P=0.029\right)$ and IL-2 $\left(r^{2}=0.23 ; P=0.009\right)$. After adjusting for $L D L$ levels, there was a statistically significant inverse relationship between plasma CoQ10 levels and levels of VCAM $\left(r^{2}=0.24 ; P=0.01\right)$ (Figure 3) and IL-10 ( $\left.r^{2}=0.24 ; P=0.02\right)$.

Conclusions: CoQ10 levels are significantly lower in septic shock patients than in healthy controls. CoQ10 is negatively associated with vascular endothelial markers and inflammatory molecules, though this association diminishes after adjusting for LDL levels.

\section{Introduction}

Severe sepsis and septic shock are major causes of morbidity and mortality. The annual incidence of deaths due to severe sepsis and septic shock is estimated to be over 215,000 in the United States [1]. Organ dysfunction related to sepsis is associated with significant alterations

\footnotetext{
* Correspondence: mdonnino@bidmc.harvard.edu

'Department of Emergency Medicine, Beth Israel Deaconess Medical Center,

One Deaconess Road, West Clinical Center 2, Boston, MA 02215, USA

Full list of author information is available at the end of the article
}

in metabolic pathways and cellular function [2]. Although the exact mechanism by which sepsis leads to organ dysfunction remains unclear, hypoxia and several mediators in the systemic inflammatory response have been shown to directly impair mitochondrial function and may lead to increased morbidity and mortality in patients with septic shock [3-5].

Coenzyme Q (CoQ10), or ubiquinone, is located within the inner mitochondrial membrane and acts as an electron transport mediator from complex I or

\section{Biomed Central}


complex II to complex III [2]. While a small portion of CoQ10 may be obtained from dietary sources (including food and oral supplementation), it is primarily synthesized endogenously in the endoplasmic reticulum from tyrosine and mevalonate and vitamins $B_{2}, B_{9}, B_{12}$ and $C$ and is transported in the plasma by low-density lipoprotein (LDL) [6]. Low CoQ10 levels may result from impairment in CoQ10 synthesis, decreased dietary intake or increased requirement (such as during conditions that increase oxidative stress), or from any combination of these factors. Previous investigations have demonstrated that prolonged statin use may decrease CoQ10 levels in patients with cardiovascular disease; however, a causal effect of stain therapy on decreased CoQ10 levels remains a topic of debate $[7,8]$. In addition to the potential negative effect that statins may have on CoQ10 levels, the increased metabolic demand resulting from sepsis may also deplete CoQ10 levels.

Statins may hold therapeutic value for the prevention and/or treatment of patients with sepsis. Previous investigations have demonstrated important immunomodulatory activity of statins on the inflammatory response and direct alterations of leukocyte-endothelial cell interactions [9-11]. Furthermore, statin therapy has been associated with decreased morbidity and mortality in patients with sepsis [12]. However, statins may also decrease levels of CoQ10, an essential component of the respiratory chain, leading to mitochondrial dysfunction [13].

The statin, CoQ10 and sepsis triangulation was recently reviewed, and the lack of human data to support theoretical assumptions and hypotheses was noted. Specifically, Brealey et al. [14] stated that low levels of antioxidants cause mitochondrial dysfunction in human sepsis, but there are currently no data to account for the levels of the mitochondrial component CoQ10. Herein we report on the levels of plasma CoQ10 in patients with septic shock and the relationship between CoQ10 and the inflammatory cascade. The primary goal of this investigation was to determine whether patients with septic shock have decreased plasma CoQ10 compared to healthy controls. Our secondary aims were to determine whether CoQ10 levels are associated with the sepsis-induced inflammatory response and vascular endothelial activation and to determine whether acute statin therapy reduces CoQ10 levels [15-19]. We measured $\mathrm{CoQ} 10$ levels and inflammatory and vascular endothelial biomarkers in plasma that had been collected in a prospective, randomized trial of statins versus placebo for patients with septic shock.

\section{Materials and methods Study design}

We conducted a post hoc analysis of a prospective, randomized trial of septic shock patients admitted to an urban university teaching hospital (ClinicalTrials.gov ID: NCT00676897). The initial trial was a prospective, randomized, double-blind, placebo-controlled, single-center trial of the effect of statin therapy for patients with septic shock. Patients enrolled in the trial were given either 40 $\mathrm{mg}$ of simvastatin or placebo once daily for a maximum of seven days or until hospital discharge, whichever came first. The primary outcome for the initial trial was the time until shock reversal with a secondary outcome of attenuation of the inflammatory cascade following intervention. The current post hoc analysis was performed using clinical and inflammatory biomarker data collected from the patients who were included in the initial trial. The study was approved by the Institutional Review Board at Beth Israel Deaconess Medical Center.

\section{Study setting and population}

All patients who presented to our urban university teaching hospital with suspected or confirmed infection were considered for enrollment. Patients were screened by trained research assistants using the hospital's electronic medical record system in the emergency department and ICUs. Inclusion criteria for the initial trial consisted of adult patients (age $>18$ years) with two or more of the four systemic inflammatory response syndrome criteria, suspected or confirmed infection (pneumonia, sepsis, pyelonephritis, and so on) and evidence of shock (defined as the use of vasopressor medication for a minimum of one hour). Exclusion criteria consisted of statin or digoxin use upon presentation, alanine aminotransferase (ALT) and aspartate aminotransferase (AST) levels more than three times the upper limit of normal or creatine phosphokinase level more than four times the upper limit of normal. Patients were also excluded if they did not require vasopressor support. Healthy controls were enrolled if they met the inclusion criteria of age $>18$ years and no acute medical or surgical problems that would require hospital admission. Controls were excluded if they had any significant comorbid disease. Written informed consent was obtained from the patients or their designated surrogates prior to any research intervention.

\section{Interventions}

All patients received standard medical therapy for the treatment of the underlying infection as determined by the clinical team. The initial randomized trial included provision of either simvastatin $40 \mathrm{mg}$ or placebo once daily for a maximum of seven days or until hospital discharge, whichever occurred first. Patients included in the post hoc analysis were assessed for clinical and biomarker data, including levels of CoQ10 and plasma LDL as well as vascular endothelial and inflammatory markers. 


\section{Data collection}

Pertinent patient demographics, including age, gender, race, medical history and suspected source of infection were recorded at baseline. Vital signs and laboratory data, including complete blood count, electrolytes and lactate, were recorded at the time of enrollment and at serial time intervals.

Blood was collected at serial time intervals (0, 24, 48, 72 and 168 hours) into evacuated tubes containing ethylenediaminetetraacetic acid from an existing venous or arterial catheter. The blood was immediately centrifuged at $2,800 \times g$ at $4^{\circ} \mathrm{C}$ for 10 minutes, and $0.5 \mathrm{~mL}$ of plasma were transferred into cryogenic vials and stored at $-80^{\circ} \mathrm{C}$ immediately after centrifugation. Samples from patients who had blood collected at both 0 and 72 hours were used in the current biomarker analysis.

\section{Primary data analysis \\ Cytokine and soluble cardiovascular biomarker measurement}

Plasma from patients and controls at 0 and 72 hours was collected and stored at $-80^{\circ} \mathrm{C}$ until thawed to assay for cytokines and soluble cardiovascular biomarkers. Analytes were measured by multiplex analysis (Bio-Plex Laboratories, Hercules, CA, USA) using 96-well multiplex kits (Millipore, Billerica, MA, USA). Analytes measured were IL-1ra, IL-2, IL-6, IL-8, IL-10, TNF- $\alpha$, vascular endothelial growth factor (VEGF), soluble Eselectin, soluble vascular cell adhesion molecule (VCAM) and soluble intercellular adhesion molecule (ICAM). For vascular biomarkers (E-selectin, VCAM and ICAM), plasma samples were measured at either 1:50 or 1:100 dilution factor. For cytokines and VEGF plasma, samples were measured undiluted. All samples were measured in duplicate (mean values from duplicate results were used for analysis).

\section{Plasma coenzyme Q10 measurement}

Plasma CoQ10 levels were measured at the Laboratories of Metabolic and Mitochondrial Diseases at Columbia University, where analysis of CoQ10 is performed for both commercial and research purposes. Concentrations of CoQ10 were measured by using high-pressure liquid chromatography (HPLC) with an electrochemical detection system. Briefly, $50 \mu \mathrm{L}$ of thawed plasma were mixed in an Eppendorf tube with $50 \mu \mathrm{L}$ of ethanol solution containing $25 \mathrm{ng}$ of CoQ9 (used as internal standard). After the addition of $900 \mu \mathrm{L}$ of 1-propanol, the tube was vortex-mixed for 2 minutes and then centrifuged at high speed in the cold room for 10 minutes. An aliquot (about $300 \mu \mathrm{L}$ ) of the supernatant was filtered through a $0.2-\mu \mathrm{m}$ filter column and transferred into an HPLC sample injection vial, and an aliquot of $50 \mu \mathrm{L}$ of the extract was injected into an HPLC system equipped with a C18 reversed-phase column and an ESA
Coulochem II electrochemical detector (ESA, Inc., Chelmsford, MA, USA). The established healthy control CoQ10 concentration range in the literature is 1.04 $\mu \mathrm{mol} / \mathrm{L} \pm 0.33$, though we measured a set of healthy controls in this study to provide direct comparison and validate our methods [20].

\section{Statistical methods}

Basic demographics and patient characteristics are reported using descriptive statistics. Continuous data are reported as medians with interquartile ranges (IQRs), and categorical data are reported as frequency with percentages. As LDL is the primary transport molecule for CoQ10, the ratio of CoQ10 to LDL (CoQ10:LDL) levels was calculated, and these values were used for subsequent analyses. CoQ10 levels in the septic population were compared to healthy controls using the Wilcoxon rank-sum test. The relationship between CoQ10 and inflammatory and vascular endothelial biomarkers was assessed using either the Pearson or Spearman correlation, depending on the normality of the data. Statistical analysis was performed using SAS version 9.2 software (SAS Institute, Cary, NC, USA), and $P<0.05$ was considered significant for statistical testing of the data.

\section{Power calculation}

Miles et al. [20] previously reported healthy control values for CoQ10 (1.04 $\mu \mathrm{mol} / \mathrm{L} \pm 0.33)$. Thus, assuming a $30 \%$ lower value in septic shock patients with a similar standard deviation, our study has $80 \%$ power to detect a difference between groups $(\alpha=0.05)$ with a sample size of 28 septic patients and 16 controls.

\section{Results}

A total of 18 subjects were randomized in the initial trial. Of these 18 patients, one patient died prior to the 72-hour follow-up examination and three had no follow-up blood specimen available and were therefore excluded from the current analysis. Of the 18 patients in the original trial, 14 had CoQ10 levels measured at both 0 and 72 hours, and these 28 samples were included in our analysis (Figure 1). The baseline characteristics of these 14 patients are provided in Table 1 . In addition, we measured CoQ10 levels in 16 healthy controls. The median age of the septic shock patients was 55 years (IQR 47 to 62 years), and $64 \%$ of them were females. The severity of illness was assessed using the Acute Physiology and Chronic Health Evaluation II (APACHE II) score, with a median score of 19 (IQR 17 to 21). There was no difference in severity of illness between groups in the initial trial on the basis of APACHE II scoring (placebo: 19 (IQR 18 to 20), statin: 17 (IQR 16 to 21 )) or lactic acid (placebo: $2.0 \mathrm{mmol} / \mathrm{L}$ (IQR 1.9 to 2.2 ), statin: $2.3 \mathrm{mmol} / \mathrm{L}$ (IQR 1.3 to 3.3 )).

CoQ10 levels for the patients were low, with a median of $0.49 \mu \mathrm{mol} / \mathrm{L}$ (IQR 0.26 to 0.62 ). The median LDL 


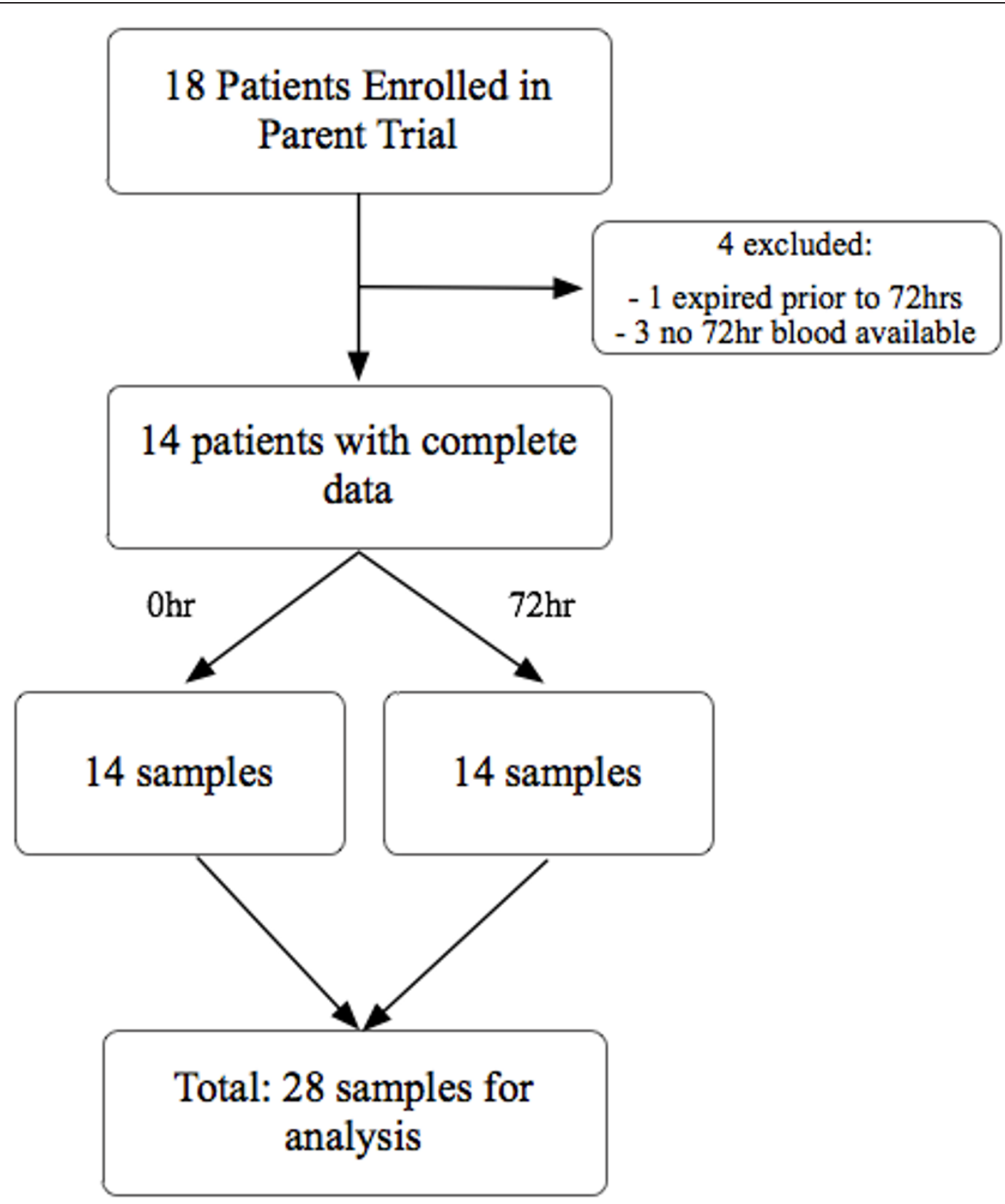

Figure 1 Schematic of our study design. A total of 28 samples were analyzed (14 patients with baseline and follow-up blood specimens were included for analysis).

level in the sample was $42 \mathrm{mg} / \mathrm{dL}$ (IQR 35 to 61), and the CoQ10:LDL ratio was 101 (IQR 82 to 150 ). CoQ10 levels in the patient group were statistically significantly lower than in the 16 healthy controls $(0.95 \mu \mathrm{mol} / \mathrm{L} \pm$ 0.29; $P<0.0001$ ). Of note, our control values of CoQ10 were similar to previously established healthy control ranges, thus validating our methods $(1.04 \mu \mathrm{mol} / \mathrm{L} \pm 0.33$ $\mu \mathrm{mol} / \mathrm{L}$ ) [20]. Randomization from the initial trial had no effect on CoQ10 level (Figure 2). Specifically, there was no significant difference in the change in mean
CoQ10 levels between randomization groups $(P=0.13)$, though there appears to have been a slight trend toward decreases in the statin group. There was a statistically significant relationship between CoQ10 level and $\operatorname{VCAM}\left(r^{2}=0.2 ; P=0.008\right)$, TNF- $\alpha\left(r^{2}=0.28 ; P=\right.$ $0.004)$, IL-8 $\left(r^{2}=0.21 ; P=0.015\right)$, IL-10 $\left(r^{2}=0.18 ; P=\right.$ $0.025)$, E-selectin $\left(r^{2}=0.17 ; P=-0.03\right)$, IL-1ra $\left(r^{2}=\right.$ $0.21 ; P=0.014)$, IL-6 $\left(r^{2}=0.17 ; P=0.029\right)$ and IL-2 $\left(r^{2}\right.$ $=0.23 ; P=0.009)$. After adjusting for LDL levels, there were statistically significant inverse relationships 
Table 1 Baseline patient characteristics ${ }^{a}$

\begin{tabular}{|c|c|}
\hline Demographics & Data $(N=14)$ \\
\hline Age, years & $55(47-62)$ \\
\hline Females, $n(\%)$ & $9(64)$ \\
\hline \multicolumn{2}{|l|}{ Race, $n(\%)$} \\
\hline African-American & $3(21)$ \\
\hline Caucasian & $11(79)$ \\
\hline \multicolumn{2}{|l|}{ Comorbidities, n (\%) } \\
\hline Diabetes & $2(14)$ \\
\hline Hypertension & $8(57)$ \\
\hline Chronic obstructive pulmonary disease & $2(14)$ \\
\hline Coronary artery disease & $2(14)$ \\
\hline Congestive heart failure & $3(21)$ \\
\hline Renal insufficiency or end-stage renal disease & $4(29)$ \\
\hline \multicolumn{2}{|l|}{ Initial vital signs } \\
\hline Temperature ( $\left.{ }^{\circ} \mathrm{F}\right)$ & $98.9(97.0-100.0)$ \\
\hline MAP (mmHg) & $64(54-83)$ \\
\hline Heart rate (bpm) & $99(88-112)$ \\
\hline Respiratory rate (breaths/minute) & $20(16-23)$ \\
\hline \multicolumn{2}{|l|}{ Initial laboratory data } \\
\hline Sodium (mEq/L) & $140(137-140)$ \\
\hline Potassium (mEq/L) & $3.8(3.1-4.2)$ \\
\hline Bicarbonate (mEq/L) & $19(16-24)$ \\
\hline Creatinine (mg/dL) & $1.5(0.9-3.5)$ \\
\hline INR & $1.2(1.2-1.5)$ \\
\hline AST (IU/L) & $29(7-35)$ \\
\hline ALT (IU/L) & $29(19-45)$ \\
\hline White blood cells $\left(1 \times 10^{3} / \mu \mathrm{L}\right)$ & $10.5(6-16)$ \\
\hline Hematocrit (\%) & $31(29-34)$ \\
\hline Platelets $\left(1 \times 10^{3} / \mu \mathrm{L}\right)$ & $196(164-302)$ \\
\hline \multicolumn{2}{|l|}{ Arterial blood gas } \\
\hline $\mathrm{pH}$ & $7.36(7.29-7.42)$ \\
\hline $\mathrm{PaCO}_{2}(\mathrm{mmHg})$ & $38(30-51)$ \\
\hline $\mathrm{PaO}_{2}(\mathrm{mmHg})$ & $101(75-113)$ \\
\hline Lactate (mmol/L) & $2.1(1.4-2.4)$ \\
\hline \multicolumn{2}{|l|}{ CoQ10 data } \\
\hline CoQ10 ( $\mu \mathrm{mol} / \mathrm{L})$ & $0.49(0.26-0.62)$ \\
\hline LDL (mg/dL) & $42(35-61)$ \\
\hline CoQ10:LDL ratio & $101(82-150)$ \\
\hline
\end{tabular}

${ }^{a}$ ALT: alanine aminotransferase, AST: aspartate aminotransferase, CoQ10: plasma coenzyme Q10, INR: International Normalized Ratio, LDL: low-density lipoprotein, MAP: mean arterial pressure, $\mathrm{PaCO}_{2}$, partial pressure of carbon dioxide, $\mathrm{PaO}_{2}$ : partial pressure of oxygen. Data are medians with interquartile ranges unless otherwise indicated.

between CoQ10 and VCAM $\left(r^{2}=0.24 ; P=0.01\right)$ (Figure $3)$ and IL-10 $\left(r^{2}=0.24 ; P=0.02\right)$. Additional biomarkers demonstrated no association with CoQ10 after adjustment for plasma LDL (all $P>0.05$ ).

\section{Discussion}

To the best of our knowledge, this is the first report on the levels of CoQ10 in humans with septic shock. In this post hoc analysis of a prospective, randomized trial,

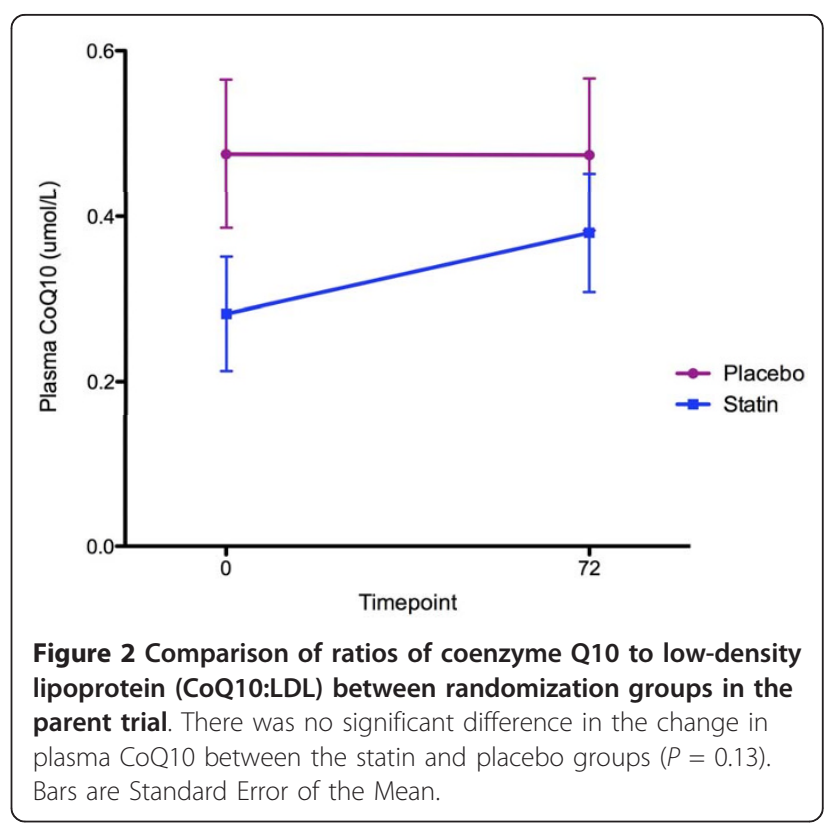

we found that patients in septic shock have significantly lower levels of plasma CoQ10 compared to a normal healthy control population [20]. Furthermore, we found a statistically significant inverse relationship between plasma CoQ10 levels with inflammatory cytokines and vascular endothelial biomarkers.

CoQ10 is an essential component of the electron transport chain, acting as an electron transporter of mitochondrial complexes I and II to complex III. Disruption of this mechanism can compromise the oxidative capacity of the mitochondria and lead to a decreased level of energy production. Mitochondria are the primary organelles for cellular energy production, and mitochondrial dysfunction can be detrimental in critical illness. The presence of mitochondrial

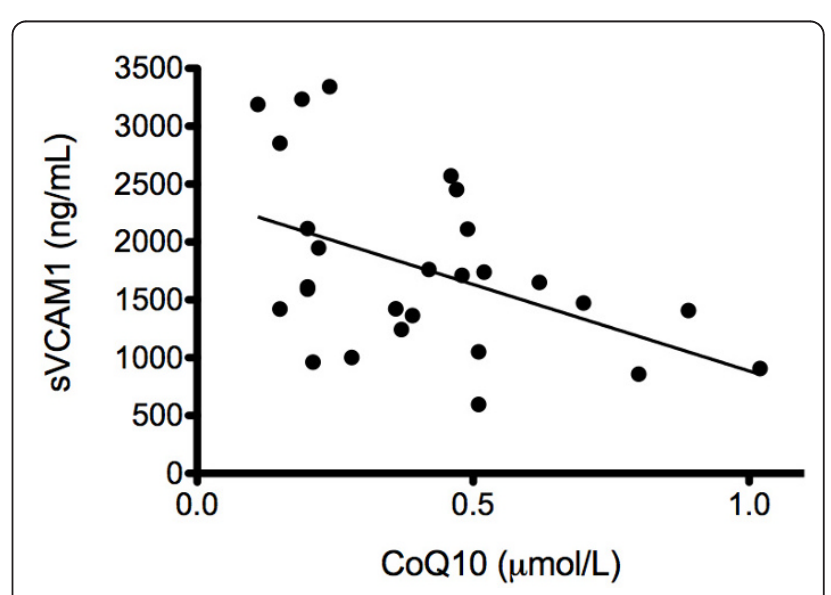

Figure 3 Association between CoQ10 and sVCAM1, soluble vascular cell adhesion molecule levels $(P=0.04)$. 
dysfunction in septic shock has been described previously, though the exact components and reasons for this dysfunction are not completely known [3-5]. The hypothesis that CoQ10 deficiency contributes to mitochondrial dysfunction remains plausible. Our findings that plasma CoQ10 levels are abnormally low in a septic shock cohort compared to healthy adults supports this potential link.

Previous research studies have demonstrated that inflammatory cytokines and vascular endothelial markers are abnormally elevated in septic shock, indicating both inflammation and activation of the vascular endothelium. Within septic patients, elevation of these biomarkers has been associated with worse outcomes [15-18]. We found a statistically significant association between CoQ10 levels and VCAM and IL-10; whether the relationship is causal remains unknown. However, CoQ10 treatment in other diseases, such as breast cancer, did show that provision of the compound ultimately resulted in a lowering of biomarkers, thus supporting a causal relationship [21].

The finding of abnormally low CoQ10 levels is consistent with the expected pathophysiology in septic shock. Since LDL is the primary transport molecule for CoQ10 in plasma, we measured LDL levels and found that these levels were low, which is in agreement with reports in the literature on lipoprotein variations in sepsis and septic shock [22]. The decrease in the carrier may lead to depletion of CoQ10. In addition, CoQ10 is partially provided by diet, and patients with critical illness often have nutritional deficiencies for multiple reasons. Furthermore, through a series of complex biochemical reactions, CoQ10 is synthesized endogenously from a number of components, including mevalonate and tyrosine as well as vitamins $B_{2}, B_{9}, B_{12}$ and $C$. Patients in septic shock have deficiencies in micronutrients, including B vitamins, and since CoQ10 in the body is primarily derived from endogenous production, metabolic dysfunction leading to decreased production and/ or increased utilization is more likely to account for CoQ10 depletion identified in this study [23]. Regardless of etiology, identifying low CoQ10 levels in patients with septic shock is significant, as the compound is essential to mitochondrial function and may play an important role in the pathophysiology of mitochondrial dysfunction in sepsis. In contrast to previous investigations of mitochondrial dysfunction, our finding that CoQ10 is low in sepsis opens the potential for future therapeutic interventions, as CoQ10 can be administered exogenously.

In experimental models, pretreatment with CoQ10 before induction of sepsis restored hepatic ATP levels, suppressed markers of lipid peroxidation and increased survivability [2]. In a canine model of septic shock, Lelli et al. [24] found that pretreatment with CoQ10 led to improvements in cardiac output and mean arterial pressure, though they did not find statistically significant differences in several biomarkers, including TNF- $\alpha$ and IL6. However, they had a very small sample size (10 dogs, 5 in each group), and they indicated that IL-6, for instance, increased by approximately $3,000 \%$ over the baseline level (prior to injection with Escherichia coli) compared to an approximately $500 \%$ increase in the group that was infused with $\mathrm{CoQ} 10$ in addition to $E$. coli, even though this did not reach statistical significance with such a small sample size. A relationship might have been found with a larger sample. In addition, there were multiple markers measured in our study that were not measured by Lelli and colleagues [24]. Thus, the improvements in cardiovascular function that these investigators found may be linked to modifications of vascular or inflammatory biomarkers.

We detected no significant effect of statin therapy on plasma CoQ10 levels in our population; however, our sample size was too small to draw any definitive conclusions, and we did note a trend toward decreases in the statin group as compared to placebo. In 1993, Watts et al. [25] reported that patients undergoing statin therapy for hyperlipidemia had significantly reduced CoQ10 levels. However, their investigation was cross-sectional in design and thus they could not determine a causal association between statin use and decreased CoQ10 levels.

The current study has several limitations which must be considered when interpreting the data. First, the sample size in our study was small. The comparison of the total data set of 28 patients to 16 healthy controls is likely robust enough to conclude that CoQ10 levels are decreased in sepsis; however the effect of statins on CoQ10 is less clear, particularly because of our small sample size. Second, there were a number of patients in septic shock who were not included in the data analysis, as they did not meet all inclusion criteria for the initial prospective, randomized trial. For example, we defined "shock" as the requirement for vasopressors for a minimum of one hour, and septic patients who were not vasopressor-dependent were not considered for enrollment in the initial trial. Therefore, larger prospective observational trials measuring CoQ10 in this patient population are required.

\section{Conclusions}

CoQ10 levels are abnormally low in patients with septic shock compared to controls. The clinical significance of this abnormality remains unknown but should be explored, given the essential role that $\mathrm{CoQ} 10$ has in the electron transport chain. Low levels of CoQ10 may be associated with high levels of inflammatory cytokines 
and vascular endothelial biomarkers, though adjusting for LDL (carrier of CoQ10) diminishes this association and may explain this finding.

\section{Key messages}

- Plasma CoQ10 levels are low in human septic shock.

- There may be an association between plasma CoQ10 levels and the inflammatory cascade in human septic shock.

- Statin therapy administered to patients in septic shock had no effect on CoQ10 levels.

- Additional research should be conducted to investigate the potential of CoQ10 as a therapeutic agent for patients in septic shock.

\begin{abstract}
Abbreviations
ALT: alanine aminotransferase; AST: aspartate aminotransferase; APACHE II: Acute Physiology and Chronic Health Evaluation II; CoQ10: coenzyme Q10; ICAM: intercellular adhesion molecule; INR: International Normalized Ratio; IL: interleukin; LDL: low-density lipoprotein; MAP: mean arterial pressure; TNF-a: tumor necrosis factor a; VCAM: vascular cell adhesion molecule; VEGF: vascular endothelial growth factor.
\end{abstract}

\section{Acknowledgements}

The authors thank Dr Peter Weller for his generous assistance with multiplex analysis. The authors also thank Francesca Montillo for her assistance in preparing the manuscript. This project was funded in part by the Eleanor Shores Grant and supported by grant UL1 RR025758-Harvard Clinical and Translational Science Center from the National Center for Research Resources. The content of this article is solely the responsibility of the authors and does not necessarily represent the official views of the National Center for Research Resources or the National Institutes of Health (NIH). Dr Praveen Akuthota is supported by NIH grant F32Al081513.

\section{Author details}

'Department of Emergency Medicine, Beth Israel Deaconess Medical Center, One Deaconess Road, West Clinical Center 2, Boston, MA 02215, USA. ${ }^{2}$ Division of Pulmonary, Critical Care and Sleep Medicine, Department of Medicine, Beth Israel Deaconess Medical Center, One Deaconess Road, Boston, MA 02215, USA. ${ }^{3}$ Trauma/Surgical/Neurological Critical Care, Department of Anesthesia Critical Care, Beth Israel Deaconess Medical Center, One Deaconess Road, Boston, MA 02215, USA. ${ }^{4}$ Department of Pathology and Cell Biology, Columbia University Medical Center, 630 West 168th Street, VC 15-208, New York, NY 10032, USA. ${ }^{5}$ Division of General Medicine and Primary Care, Department of Medicine, Beth Israel Deaconess Medical Center, 330 Brookline Ave, Boston, MA 02215, USA. ${ }^{6}$ Division of Allergy and Inflammation, Department of Medicine, Beth Israel Deaconess Medical Center, One Deaconess Road, Boston, MA 02215, USA.

\section{Authors' contributions}

MWD and MNC designed the study and were responsible for all aspects of this investigation. JS and DK were responsible for primary data collection and substantial portions of the data interpretation and analysis. PA was responsible for vascular endothelial biomarker and inflammatory cytokine assays. AN was responsible for the $\mathrm{COQ} 10$ assays. CB provided oversight on the interpretation of the CoQ10 results. All authors were involved in the writing of the manuscript and approved the final version.

\section{Competing interests}

The authors declare that they have no competing interests.

Received: 21 February 2011 Revised: 11 June 2011 Accepted: 9 August 2011 Published: 9 August 2011

\section{References}

1. Angus DC, Linde-Zwirble WT, Lidicker J, Clermont G, Carcillo J, Pinsky MR: Epidemiology of severe sepsis in the United States: analysis of incidence, outcome, and associated costs of care. Crit Care Med 2001, 29:1303-1310

2. Dare AJ, Phillips AR, Hickey AJ, Mittal A, Loveday B, Thompson N, Windsor JA: A systematic review of experimental treatments for mitochondrial dysfunction in sepsis and multiple organ dysfunction syndrome. Free Radic Biol Med 2009, 47:1517-1525.

3. Brealey D, Brand M, Hargreaves I, Heales S, Land J, Smolenski R, Davies NA, Cooper CE, Singer M: Association between mitochondrial dysfunction and severity and outcome of septic shock. Lancet 2002, 360:219-223.

4. Harrois A, Huet $O$, Duranteau J: Alterations of mitochondrial function in sepsis and critical illness. Curr Opin Anaesthesiol 2009, 22:143-149.

5. Fink MP: Cytopathic hypoxia: mitochondrial dysfunction as mechanism contributing to organ dysfunction in sepsis. Crit Care Clin 2001, 17:219-237.

6. Tomasetti M, Alleva R, Solenghi MD, Littarru GP: Distribution of antioxidants among blood components and lipoproteins: significance of lipids/CoQ10 ratio as a possible marker of increased risk for atherosclerosis. Biofactors 1999, 9:231-240.

7. Rundek T, Naini A, Sacco R, Coates K, DiMauro S: Atorvastatin decreases the coenzyme Q10 level in the blood of patients at risk for cardiovascular disease and stroke. Arch Neurol 2004, 61:889-892.

8. Hedenmalm K, Alvan G, Ohagen P, Dahl ML: Muscle toxicity with statins. Pharmacoepidemiol Drug Saf 2010, 19:223-231.

9. Kwak B, Mulhaupt F, Myit S, Mach F: Statins as a newly recognized type of immunomodulator. Nat Med 2000, 6:1399-1402.

10. Park SY, Lee JS, Ko YJ, Kim AR, Choi MK, Kwak MK, Choi HG, Yong CS, Kim JA: Inhibitory effect of simvastatin on the TNF-a- and angiotensin IIinduced monocyte adhesion to endothelial cells is mediated through the suppression of geranylgeranyl isoprenoid-dependent ROS generation. Arch Pharm Res 2008, 31:195-204.

11. Terblanche M, Almog Y, Rosenson RS, Smith TS, Hackam DG: Statins and sepsis: multiple modifications at multiple levels. Lancet Infect Dis 2007, 7:358-368.

12. Donnino MW, Cocchi MN, Howell M, Clardy P, Talmor D, Cataldo L, Chase M, Al-Marshad A, Ngo L, Shapiro NI: Statin therapy is associated with decreased mortality in patients with infection. Acad Emerg Med 2009, 16:230-234.

13. Littarru GP, Tiano L: Clinical aspects of coenzyme Q10: an update. Nutrition 2010, 26:250-254.

14. Brealey DA, Singer M, Terblanche M: Potential metabolic consequences of statins in sepsis. Crit Care Med 2011, 39:1514-1520.

15. Casey LC, Balk RA, Bone RC: Plasma cytokine and endotoxin levels correlate with survival in patients with the sepsis syndrome. Ann Intern Med 1993, 119:771-778.

16. Damas $P$, Ledoux D, Nys $M$, Vrindts $Y$, De Groote $D$, Franchimont $P$, Lamy M: Cytokine serum level during severe sepsis in human IL-6 as a marker of severity. Ann Surg 1992, 215:356-362.

17. Hack CE, De Groot ER, Felt-Bersma RJ, Nuijens JH, Strack Van Schijndel RJ, Eerenberg-Belmer AJ, Thijs LG, Aarden LA: Increased plasma levels of interleukin-6 in sepsis. Blood 1989, 74:1704-1710.

18. Pinsky MR, Vincent JL, Deviere J, Alegre M, Kahn RJ, Dupont E: Serum cytokine levels in human septic shock: relation to multiple-system organ failure and mortality. Chest 1993, 103:565-575.

19. Reinhart K, Bayer O, Brunkhorst F, Meisner M: Markers of endothelial damage in organ dysfunction and sepsis. Crit Care Med 2002, 30(5 Suppl): S302-S312.

20. Miles MV, Horn PS, Tang PH, Morrison JA, Miles L, DeGrauw T, Pesce AJ: Age-related changes in plasma coenzyme Q10 concentrations and redox state in apparently healthy children and adults. Clin Chim Acta 2004, 347:139-144.

21. Premkumar VG, Yuvaraj S, Vijayasarathy K, Gangadaran SG, Sachdanandam P: Serum cytokine levels of interleukin-1 $\beta,-6,-8$, tumour necrosis factor- $a$ and vascular endothelial growth factor in breast cancer patients treated with tamoxifen and supplemented with co-enzyme $\mathrm{Q}_{10}$, riboflavin and niacin. Basic Clin Pharmacol Toxicol 2007, 100:387-391.

22. van Leeuwen HJ, Heezius ECJM, Dallinga GM, van Strijp JAG, Verhoef J, van Kessel KPM: Lipoprotein metabolism in patients with severe sepsis. Crit Care Med 2003, 31:1359-1366. 
23. Donnino MW, Carney E, Cocchi MN, Barbash I, Chase M, Joyce N, Chou PP, Ngo L: Thiamine deficiency in critically ill patients with sepsis. J Crit Care 2010, 25:576-581.

24. Lelli JL, Drongowski RA, Gastman B, Remick DG, Coran AG: Effects of coenzyme Q10 on the mediator cascade of sepsis. Circ Shock 1993, 39:178-187.

25. Watts GF, Castelluccio C, Rice-Evans C, Taub NA, Baum H, Quinn PJ: Plasma coenzyme Q (ubiquinone) concentrations in patients treated with simvastatin. J Clin Pathol 1993, 46:1055-1057.

doi:10.1186/cc10343

Cite this article as: Donnino et al: Coenzyme 010 levels are low and may be associated with the inflammatory cascade in septic shock. Critical Care 2011 15:R189.

Submit your next manuscript to BioMed Central and take full advantage of:

- Convenient online submission

- Thorough peer review

- No space constraints or color figure charges

- Immediate publication on acceptance

- Inclusion in PubMed, CAS, Scopus and Google Scholar

- Research which is freely available for redistribution

Submit your manuscript at www.biomedcentral.com/submit 